II. POLARIZATION 


\section{POLARIMETRY AND PHYSICS OF Be STAR ENVELOPES}

(Review paper)

George V. Coyne, S.J.

University of Arizona and Vatican Observatory

Ian S. McLean

Royal Observatory Edinburgh

A review of the most recent developments in polarization studies of Be stars is presented. New polarization techniques for high-resolution spectropolarimetry and for near infrared polarimetry are described and a wide range of new observations are discussed. These include broadband, intermediate-band and multichannel observations of the continuum polarization of Be stąrs in the wavelenght interval $0.3-2.2$ microns, high resolution ( $0.5 \AA)$ line profile polarimetry of a few stars and surveys of many stars for the purposes of statistical analyses. The physical significance of the observational material is discussed in the light of recent theoretical models. Emphasis is placed on the physical and geometrical parameters of Be star envelopes which polarimetry helps to determine.

\section{INTRODUCTION}

The mere fact that the optical radiation from Be stars is observed to be intrinsically 1 inearly polarized provides perhaps our most conclusive clue that the circumstellar shells of these stars are disk-like in nature. It is not surprising then that Be stars are relatively wel1studied for polarization although, as we will demonstrate, this is a tool which still remains under-exploited.

At the time of IAU Symposium 70 on Be stars and She11 stars (Slettebak, 1976) where the last review of polarization in Be stars was presented (Coyne, 1976), polarimetry at high spectral resolutions was just in its infancy. Wide-band polarimetry at optical wavelenghts had barely provided sufficient observational material for the construction of preliminary models of the shell structure about Be stars (Capps, Coyne and Dick, 1973 ; Cassine11i and Haisch, 1974). Since 1975 a number of new polarimeters have been placed in operation so that not only has there been a dramatic increase in the data base but spectropolarimetry has also been extended into the infrared whilg, across the H-beta spectral feature, resolutions as good as $0.5 \mathrm{~A}$ have been achieved. 
Simultaneous with these developments in polarimetric observations, there have occurred a number of new approaches to the modelling of shells about Be stars which attempt to predict and account for intrinsic polarization. These have resulted, for example, in stellar wind models for Be stars which, in contrast to earlier models, take account of a star of finite size, of line and continuum emission and absorption in the circumstellar she1l, of the variation of density and temperature with distance from the star, of scattering of both stellar and circumstellar radiation and, significantly, of the kinematic properties (rotation and expansion) of the shell.

Since there is a specific review paper on atmospheric models of Be Stars to be given by Poeckert elsewhere in this symposium, we will not deal critically or at lenght with models. Rather, while reviewing the observational results, we shall emphasize those physically significant parameters of Be stars which polarimetry helps to determine or even, at times, exclusively determines.

First we present a brief summary of the intrumental developments that have affected substantially the accumulation of observational data on the polarization of Be stars. Then, after a review of observational material acquired since 1975, we discuss the physical significance of the new results in terms of general physical principles and some currently proposed models for Be star shells.

\section{INSTRUMENTAL DEVELOPMENTS}

Since 1975 the principal instrumental developments which have directly contributed to Be star studies have been the diversification and progress in techniques of high resolution spectropolarimetry at optical wavelenghts and improvements of IR polarimeters. McLean (1980) has recently reviewed modern polarimetric techniques. Here we present only the highlights of those techniques which have been specifically applied to measurements of Be stars.

Already in 1973 at the $5 \mathrm{~m}$ Hale telescope, Landstreet and Angel had used a multichannel spectrophotometer (the Oke Scanner) with a Pockels cell and Glan-air prism before the entrance aperture (Angel and Landstreet, 1974) to gbtain polarization measunements of Gamma Cas at resolutions of 20-40 A across the entire visible spectrum. Those results were not published until later (Poeckert and Marlborough, 1978a) As of 1975 several polarimeters, employing the technique of scanning across atomic lines by tilting a narrow-band interference filter, had been used successfully to measure polarization sequentially at discrete wavelength intervals across the hydrogen emission lines (H-alpha and $\mathrm{H}$-gamma) ; resolutions of 2 to $12 \mathrm{~A}$ were achieved. Such polarimeters and their earlier results were discussed in the review by Coyne (1976). Tilt-scanning polarimeters have continued to be extremely useful since 1975 in the study of many emission-line stars and binaries, e.g. Beta 
Lyrae (McLean, 1977), a detailed study of H-alpha in Gamma Cas (Poeckert and Marlborough, 1977), and a survey of southern hemisphere Be stars (McLean and Clarke, 1979). Poeckert, Bastien and Landstreet (1979) have employed a set of narrow-band filters to survey a large number of $\mathrm{Be}$ stars, whereas Hayes (1978) has used a broad-band technique to search for intrinsic polarization in 0-type emission-1ine stars. All of the above polarimeters use a photomultiplier tube as the detector and, with the exception of the Oke Scanner, all of the measurements are made sequentially.

More recently, attention has focussed on the application to spectropolarimetry of new multi-element photoelectric detectors which, coupled to spectrographs, permit simultaneous measurements at many wavelengths with high spectral resolution.

The highest spectral resolution polarimetry to date has been obtained by McLean et al. (1979) with a Digicon echelle-spectropolarimeter. Resolutions of $0.5 \mathrm{~A}$ across the $\mathrm{H}$-beta 1 ine of several $\mathrm{Be}$ and shell stars have been obtained. In that instrument the polarization modulator consisted of a superachromatic halfwave plate continuously rotating at $3.5 \mathrm{~Hz}$ and followed by a Glan prism. An echelle spectrograph in an off-plane configuration forms a spectrum on the photocathode of a Digicon image tube where photoelectrons emitted by the photocathode are then focussed to form an electron image on a linear array of 106 diodes. Another Digicon (Beaver et a1. 1972) has been used with a Pockels cell and a Cassegrain spectrograph at the University of Arizona (Stockman et a1. 1979) for the measurement of circular polarization due to the Zeeman effect. Unfortunately, these Digicons had a fairly limited life span and are no longer commercially available. Some, however, are being manufactured under contract to the Space Telescope. Other multichannel devices which have proved successful with polarimeters but which have not yet been applied to Be stars include Reticon self-scanning linear photodiode arrays and image intensifier dissector scanners (IDS). Two IDS systems, both employing Pockels cells, are in use, one at University of Western Ontario (Tomaszewski, Landstreet and use, one at University of 1980) and one at Lick Observatory (Miller, Schmidt and Robinson 1980). More recently there has been a move towards intensified Vidicons and image photon counting devices as well as towards solid state sensors such as Charge Injection and Charge Coupled Devices.

New developments have also occurred in infrared polarimetry. For example, beat frequency photo-elastic modulators (Kemp et at. 1977) have been introduced for the 1-10 micron range, although they have so far been little used for Be star studies. Jones (1979) introduced improvements into an existing IR polarimeter by placing the rotating polarization analyzer in front of all the beam splitting and beam directing mirrors and by using a quartz Lyot depolarizer to reduce any spurious effects introduced by the mirror. This instrument has been used to obtain the polarization of Be stars at wavelengths of 1.25 and 2.2. microns. 


\section{OBSERVATIONAL RESULTS}

Over the past six years the principal contributions to the polarimetric data on Be stars have been : (1) further and higher spectral resolution measurements of polarization across the hydrogen emission lines ; (2) additions to the meagre data prviously available in the infrared ; (3) more information, for more stars, on polarization variability both in wide bands and at high spectral resolution ; (4) statistical analyses of polarization in comparison with other parameters.

Before reviewing the new observations, it is useful to know that in almost all cases it is possible, by a careful combination of techniques, to remove the contaminating effects due to interstellar polarization. Only then can the intrinsic polarization be modelled in detail. In ge neral these techniques consist in : (1) comparison of the magnitude and directions of the polarizations for other stars at about the same distance and in the same direction as the program star ; (2) variations in the observed polarization and/or position angle of the plane of polarization with time and wavelength ; (3) variation of the observed polarisation across spectral lines when the effect of the line is known. Recent applications of these techniques have been presented by McLean and Clarke (1979) who surveyed the fifteen brightest southern Be stars, separated the intrinsic and interstellar components and discussed the possible difficulties and misuse of the methods of analysis. A numerical method based on technique (2) above has also been applied to $70 \mathrm{Be}$ stars by Poeckert, Bastien and Landstreet (1979).

The reduced polarization observed in the lower Balmer emission lines of a few bright Be stars was found to be approximately inversely related to the emission-line strengths, hence implying that the emission line flux was essentially unpolarized (cf. Coyne, 1979). Using a twochannel Pockels cell polarimeter, Poeckert and Marlborough (1976) obtained additional polarization measures for a number of Be stars in the $\mathrm{H}-\mathrm{alpha}$ line and neighbouring continuum and, employing the inverse relationship mentioned above, they direved intrinsic polarizations and correlated these with Vsini. Already, however, improvements in the observations were beginning to reveal evidence that a simple unpolarized emission model was inadequate. "Filling-in" of the line polarization was reported for Phi Per by Coyne and Mc Lean (1975) and variations or oscillations in position angle across the emission lines of Beta Lyrae were observed by McLean (1977). Poeckert and Marlborough (1977) made polarimetric scans across the $\mathrm{H}-\mathrm{al}$ pha 1 ine in Gamma Cas using an interference filter with $1.4 \AA$ full width at half-maximum (FWHM) tuned to an accuracy of $0.5 \AA$. Those observations clearly indicated that the decrease of polarization at the line centre could not be attributed solely to unpolarized emission. Small variations in the position angle of the linear polarization across the line were observed which, because of their antisymmetry with respect to the line centre, could not be the product of interstellar polarization but must in fact result from the rotation of a disk-1ike envelope. 
While monitoring the continuum polarization in a band near $\mathrm{H}-\mathrm{alpha}$ variations in the polarization amounting in some cases to 0.1 percent in a day were observed. Furthermore, changes with time in the position angle of the line polarization were detected and the largest such changes seem to occur when $V / R$ of the emission-line profile is not close to unity.

Using the same techniques, Poeckert and Marlborough (1978a) measured simultaneously the polarization in two intermediate continuum bands located on either side of the Balmer discontinuity. These date were then combined with their previous $\mathrm{H}$-alpha data and with polarimetric scans with Oke Scanner in the discussion of an envelope model. The combined observational data are shown in Fig. 1 where for the position angle (top) and polarization (bottom) the individual symbols represent measures made on different nights from January 1976 to January 1977. The light solid line represents the spectropolarimetry of Landstreet and Angel and the heavy solid line represents a model fit. The polarization changes abruptly across the series limits and there is also a slight position angle change. Actually, at higher spectral resolution the polarization discontinuities are not so abrupt (McLean 1981). Shortwards of the Balmer absorption edge the polarization remains nearly constant with time while that in all other bands changed. There is a sharp increase with time in the linear polarization in all measured bands but especially in the continuum just longwards of the Balmer limit where, in about five months, the polarization increased by 0.3 percent. The authors find that this is correlated with an increase in the equivalent width of the H-alpha line in emission and with an increase in the $V / R$ asymmetry of the line profile.

Employing a two-channel, rotating-waveplate polarimeter with tiltscanning of narrow-band interference filters, McLean and Clarke (1979) measured the flux and polarization across $\mathrm{H}-\mathrm{alpha}(8.5 \AA \mathrm{FWHM})$ and H-beta ( $2.3 \AA \mathrm{FWHM})$ and at four continuum wavelengths in most of the Southern hemisphere $B e$ and shell stars brighter than about fifth magnitude. For stars with extremely strong emission such as Alpha Ara and Delta Cen, the line center polarization was not as weak as might be expected from unpolarized emission and the shell star Eta Cen, which was undergoing an outburst, actually exhibited an increase of polarization at H-beta as well as a change of continuum polarization of 0.2 percent in two weeks. These results argue that the emission line flux is itself partly polarized and/or that there is further shell absorption in the line of direct unpolarized starlight. For several stars they found that the intrinsic polarization position angles are variable, indicating a non-axisymmetric distribution of scattering material which may be due to a binary character for these stars.

Recently McLean (1981) has discussed polarization observations of Zeta Tau made with 20-50 \& resolution across the Balmer jump using the single channel spectropolarimeter of the Royal Observatory Edinburgh. These observations, like the data for Gamma Cas, reveal that the 
intrinsic polarization is remarkably stable just shortward of the Balmer jump but that it varies markedly with time in the Paschen continuum. Also, the expected abrupt discontinuity in polarization at the Balmer limit is considerably "rounded". This is due undoubtedly to the confluence of the Balmer lines as the series limit is approached.

The highest spectral resolution polarimetry to date was obtained across the H-beta 1 ine for four Be stars by Mc Lean, Coyne, Frecker and Serkowski (1979) with the Digicon Echelle spectropolarimeter described earlier. A spectral resolution of about $0.5 \AA$ was obtained by averaging over 1.5 diodes ( 3 channels) of the 106 element (212 channe1) array. The results are displayed in Fig. 2 where for each star there is presented from top to bottom, the position angle of the polarization plane, the percent linear polarization and the normalized intensity profile across the H-beta line. While there are significant differences from one star to the next the following common features are present : (1) the linear polarization decreases as one approaches the line center from the wings and the decrease is not usually symmetrical for the two wings ; (2) the linear polarization increases at the line center and this generally associated with a central absorption core in the line profile; (3) secondary minima in the linear polarization occur in the line wings; (4) there are complex variations in the position angle across the lines which in general are neither symmetric nor antisymmetric with respect to the line center, although there is a tendency toward antisymmetry associated with certain localised turning points in the intensity profiles. The physical significance of these these new observations is discussed in the next section.

As of 1975 linear polarization measurements at wavelengths greater than one micron existed for only two stars, Phi Per (Coyne and Mc Lean 1975) and Zeta Tau (Capps, Coyne and Dick, 1973). Jones (1979) has published polarization observations at 1.25 and 2.2 microns for eight Be stars and has used his data to improve upon previously existing models of the continuum flux and polarization.

There have three noteworthy observational surveys and statistical analyses of the polarization in Be stars. Poeckert and Marlboroagh (1976) determined the intrinsic linear polarization for 48 Be stars from $\mathrm{H}-\mathrm{al}$ pha line-to-continuum measurements and compared these with Vsini in the context of a specific, numerically calculated model. McLean and Brown (1978), using the methods discussed by Mc Lean and Clarke (1979), analysed the existing data on 67 Be stars and derived intrinsic polarizations. On the basis of general physical considerations they derived an analytic expression relating intrinsic polarization and the inclination of the stellar rotation axis which is valid for all optically thir axisymmetric Be star envelopes (cf. Brown and Mc Lean, 1977). 


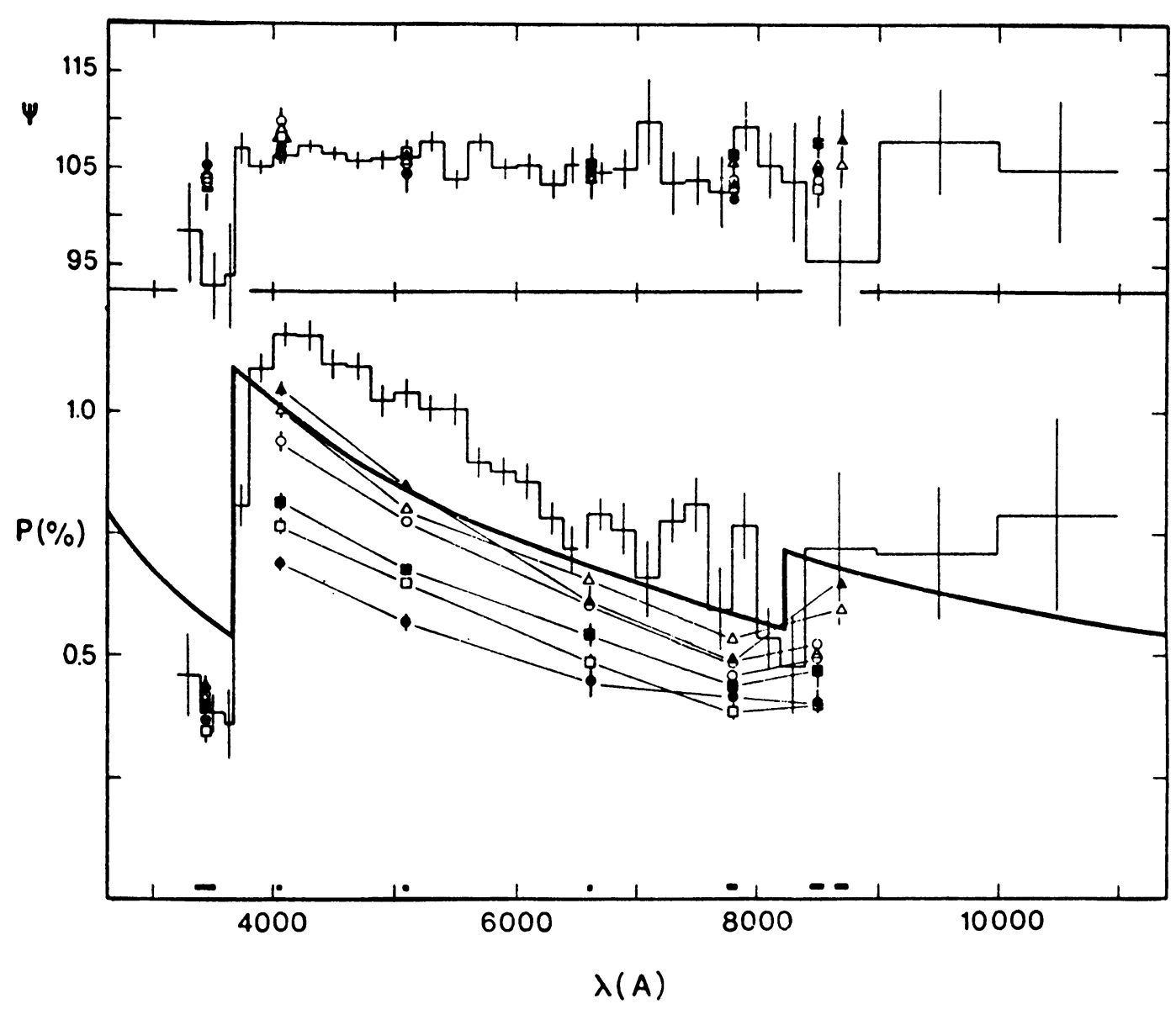

Fig. 1. - Position angle of polarization (top) and percent polarization (bottom) versus wavelength for Gamma Cas. The solid line represents a model fit by Poeckert and Marlborough (1978a) and the thin line the observations they present from scans made by Landstreet and Angel with the Oke scanner. The other individual symbols represent observations made on different nights between January 1976 and January 1977 by Poeckert and Mar1borough (1978a).

The results of both studies may be summarised as follow : (1) the intrinsic polarization is never greater than about 2 percent indicating that extremely oblate envelope do not occur and/or direct unpolarized starlight always makes a strong contribution; (2) low values of the intrinsic polarization dominate, indicatirg that there are more objects in which the circumstellar envelopes are more nearly spherical shells 

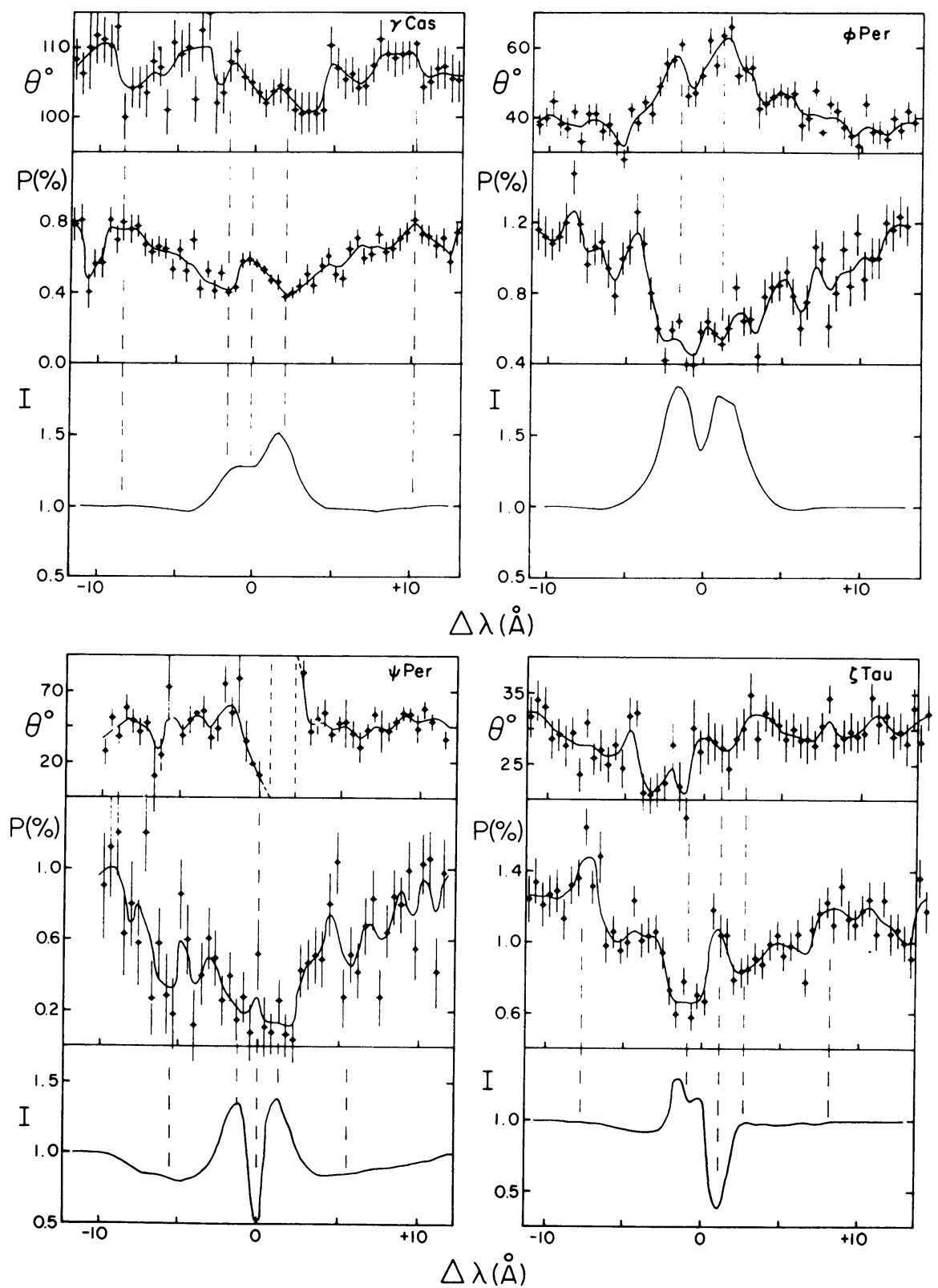

Fig. 2. For each of the four designated stars the position angle of the polarization, the percent linear polarization and the normalized intensity profile (top to bottom) across H-beta are presented. The observations at a spectral resolution of about $0.5 \AA$ were made with a Digicon Echelle spectropolarimeter (McLean et al., 1979). 
(as opposed to highly flattened disks) and/or the electron density is usually quite low; (3) there is a definite upper and lower boundary to the plot of intrinsic polarization versus rotational velocity. There are no Be stars with low rotational velocities and high polarization, which probably indicates that a certain minimal rotational velocity is required to produce a flattened shell.

Poeckert, Bastien and Lanstreet (1979) surveyed the polarization for 70 bright northern hemisphere Be stars using intermediate-band filters at the Balmer and Paschen edges and in the Paschen continuum. This survey demonstrated quite clearly that the size of the polarization discontinuities and the slope of the Paschen continuum polarization varies considerably from star to star, indicating differences of electron density and temperature among the various stellar envelopes. A number of the stars observed showed time variable polarization and, more importantly, some of them showedan apparent time lag between polarimetric and spectroscopic variations. Again, those stars with large intrinsic polarizations have large rotational velocities and strong emission 1 ines.

Another possible source of correlation is between polarization and the strength or equivalent width of the Balmer emission lines which, in a tenuous hydrogen envelope, is proportional to $\mathrm{N}_{\mathrm{e}}^{2}$. In the denser $\mathrm{Be}$ star envelopes the situation is more complex. Mc Lean (1979), from a plot of intrinsic polarization versus equivalent width of $\mathrm{H}$-alpha, finds a tendency for the most highly polarized stars to have large equivalent widths. Variations of equivalent width and changes in polarization with time have also been noticed. For instance, in Pi Aqr (North and 0lofsson, 1977, Mc Lean, 1979) both have increased, while in Gamma Cas (Poeckert and Marlborough, 1977 ; Mc Lean, 1979) both have decreased with time.

Before terminating this observational section it should be remarked that, although significant advances have been made in the past five years in the polarimetric observations of Be stars, there remains a good deal to do. This can be seen by studying the polarimetric data for 67 stars assembled by Mc Lean and Brown (1978). In that 1 ist of stars only onehalf had been measured in more than one pass-band and only one-third had repeated measurements.

\section{PHYSICAL SIGNIFICANCE OF NEW OBSERVATIONS}

It is obvious that any self-consistent model of the shells about Be stars must account for all observed parameters : the continuum flux at all wavenlengths and its variability, line profiles and their variability, continuum and line polarization and its variability, etc... Our limited purpose here, however, is to highlight those observations discussed above which provide physical insight into the nature of the shells about Be stars. 
Let us start with the now-accepted notion that the polarization in Be stars is due to scattering of the stellar radiation by free electrons in an oblate, axisymmetric envelope of ionized hydrogen about the star. This model, first proposed by Coyne and Kruszewski (1969), has been elaborated upon by Capps, Coyne and Dyck (1973) and further discussed in an analytic form, by Mc Lean (1979). Most recently it has received a more sophisticated treatment by Jones (1979). All of these models are ad hoc in the sense that physical and geometrical parameters of the shell are arbitrarily varied to try to fit the observations. On the other hand, Brown and Mc Lean (1977) provide a very useful generalized analytical treatment for the polarization resulting from single electron scattering in any axisymmetric, optically thin, envelope distribution about the star considered as a point source. By neglecting absorption and emission in the shell, they show that the resultant polarization depends on the shape of the density distribution function, the mean scattering optical depth and the inclination of the rotation axis to the line of sight. This same approach was extended by Mc Lean (1979) to include the physical effects of absorptive opacity and emission. He pointed out that, although the range of applicability of such analytic methods is limited, they are useful for gaining an insight into the physical characteristics of the envelopes. For example, the analysis of Mc Lean (1979) reveals the same dependence on the inclination of the rotation axis as that revealed by the stellar wind models of Poeckert and Marlborough (1976). Also, Daniel (1980) has recently performed Monte Carlo calculations for multiple electron scattering in Be star envelopes which, in the limit of single scattering with a small central source, approach the analytic results. Again, approximate expressions for the wavenlength dependence of polarization in an optically thin envelope containing hydrogen atoms undergoing ionizations and recombinations (Mc Lean, 1979) reveal that it is the average electron temperature, electron density and size of the envelope that control the form of the polarization curve. Suc analytic results also tend to reveal more explicitly the number of free parameters and hence the limits on uniqueness of model fits.

Turning to numerical model calculations, consider the model proposed by Jones (1979) which has the following elements : (1) the source star has a finite size and is spherical; (2) the star is surrounded by an axisymmetric flat disk of uniform thickness except that it tapers near the star ; (3) the envelope density varies with distance from the star; (4) light from the star is allowed to scatter twice before exiting from the disk ; (5) absorption and emission processes take place in the disk and the disk emission is allowed to scatter once before leaving. Again this is an ad hoc model but it is important because it introduces further sophistication into the processes producing polarization. Jones (1979) has provided a useful discussion of the dependence of various observed parameters on the physical and geometric properties of this particular disk model but much of the discussion will relate to any axisymmetric model. 
Jones (1979) discusses the application of his disk model to $7 \mathrm{Be}$ stars with the following general conclusions : (1) the same electrons that polarize by scattering also provide the IR excess by free-free emission ; (2) the electron density must increase near the star in order to explain the drop in polarization beyond 1 micron ; (3) the temperature of the scattering regions of the disk can be determined from the Balmer and Paschen continua polarizations. According to Jones, details of the spectroscopic observations may refer to other parts of the disk.

Several comments on particular stars are of interest. For Gamma Cas the inclination is $45^{\circ}$. This value allows a simultaneous fit to the overall level of polarization and the wavelength dependence. This is in good agreement with the model fit to the H-Alpha line with stellar wind calculations by Poeckert and Marlborough (1977) and is consistent with the position angle structure in the H-Beta line observed at $0.5 \AA$ resolution. Another interesting case is 48 Librae. According to Jones (1979) the disk may actually be a detached equatorial ring whose inner edge begins at 2 stellar radii. This deduction is made on the basis that a cool enough disk temperature to explain the optical polarization does not explain the infrared polarization and excess. If the disk is a ring then the IR polarization is due mainly to scattering of radiation emitted from within the ring. The proposed geometry is similar to the ring models of Hazlehurst (1967) and Huang (1972).

Totally independent constraints from those discussed above can be placed on shell models of Be stars by information derived from the photometric and polarimetric profiles of the Balmer emission 1 ines in these stars. Using a development of the Brown, Mc Lean and Emslie (1978) non- axisymmetric electron scattering models of close binaries with gas streams, Mc Lean (1979) was able to demonstrate that complex patterns of variation in line profile polarizations are to be expected in a11 envelopes undergoing large-scale mass motions irrespective of the embedded star. This follows because the severe Doppler broadening of envelope lines effectively affords a means of spatially resolving the circumstellar structure. For instance, Mc Lean (1979) has shown analytically that for a rotating star having an arbitrary axial inclination to the line of sight, since one sees deeper into the receding part of the envelope at wavelengths in the blue wing of a spectral line and deeper into the approaching part at wavenlengths in the red wing, one obtains a different spatial integration of the scattered light at different wavelengths over the spectral line and the Stokes parameters of the polarized light will vary accordingly.

In a series of papers Poeckert and Marlborough (1977, 1978a, 1978b, 1979) have developed radiative transfer models involving a stellar windelectron scattering axisymmetric envelope, including a prescription for the envelope kinematics, and have applied the model to observations principally of Gamma Cas but also Phi Per. In order to appreciate the contraints placed on such a model by the high resolution Digicon/ 
echelle H-beta observations presented in Fig. 2, it is worthwhile to review all the ways in which polarization structure may be generated in a line by processes in the envelope: (1) more or less of the direct starlight may be obscured causing respectively an increase or decrease in the polarization ; (2) polarized flux may be absorbed, hence reducing the polarization ; (3) the emission line radiation may be unpolarized or it may be scattered and polarized which will respectively decrease or increase the overall polarization at that wavelength ; (4) in a line profile dominated by Doppler broadening from mass motions in the envelope we see different parts of the polarizing envelope at different wavelengths as discussed above. Hence we see different polarization planes and different contributions of effects (1) to (3) depending on wavelength. More important1y, note that in an envelope containing differential rotation about an axis plus radial expansion plus other distortions, the structure in the position angle of polarization across the line will be complex. With these features in mind, it is possible to unterstand much of the detailed structure in the H-beta polarizations shown in Fig. 2 by following the discussion by Poeckert and Marlborough (1978a) who demonstrate the gradual "build-up", according to the stellar wind models, of the intensity profile and associated polarization parameters as the extent of the envelope is increased. The discussion centres upon Fig. 3 and Fig. 4. Various profiles of $\mathrm{H}-\mathrm{alpha}$ in Fig. 4 have been derived by cutting off the transfer solution at the radial point in the envelope indicated by the number on each profile which corresponds to the radial distance from the star centre (in units of the stellar radius) shown in Fig. 3. The extreme wings of the line in Fig. 4 are formed within $3 R_{\star}$ (where $R_{\star}$ is the stellar radius), whereas the principal line centre assymetries occur mainly between $6 R_{\star}$ and $9 R_{\star}$. This can be understood by referring to the radial-velocity contour map of the equatorial plane shown in Fig. 3. Referring to the polarization "profiles" of Fig. 4 we see that 70 percent of polarized continuum flux occures within $3 R_{\star}$. Minima in the polarization curve first appear in the wings and then move towards the line centre with increasing envelope size. These changes can be explained as follows : (1) absorption by those parts of the envelope along the observer's line of sight to the star will tend to increase the polarization while absorption at the edges of the envelope will tend to decrease the polarization. Since the envelope is wedge shaped the front part of the envelope to the observer absorbs more than the back part. Thus the polarization minima in the line wings are due to absorption at the edges of the envelope. Since the rotational velocity of the envelope is decreasing with increasing radius the absorption at the edges will have a smaller and smaller Doppler shift and the polarization minima will therefore move towards the line centre. Obviously, other velocity patterns will produce slightly different effects and the absorption process is a function of electron temperature and density. Indeed as pointed out by McLean et al. (1979), even this very detailed model does not predict all of the structure observed in the H-beta 1 ines of Gamma Cas and Zeta Tau. This is partly interpreted as indicating that the kinematical effects have been oversimplified. Note 


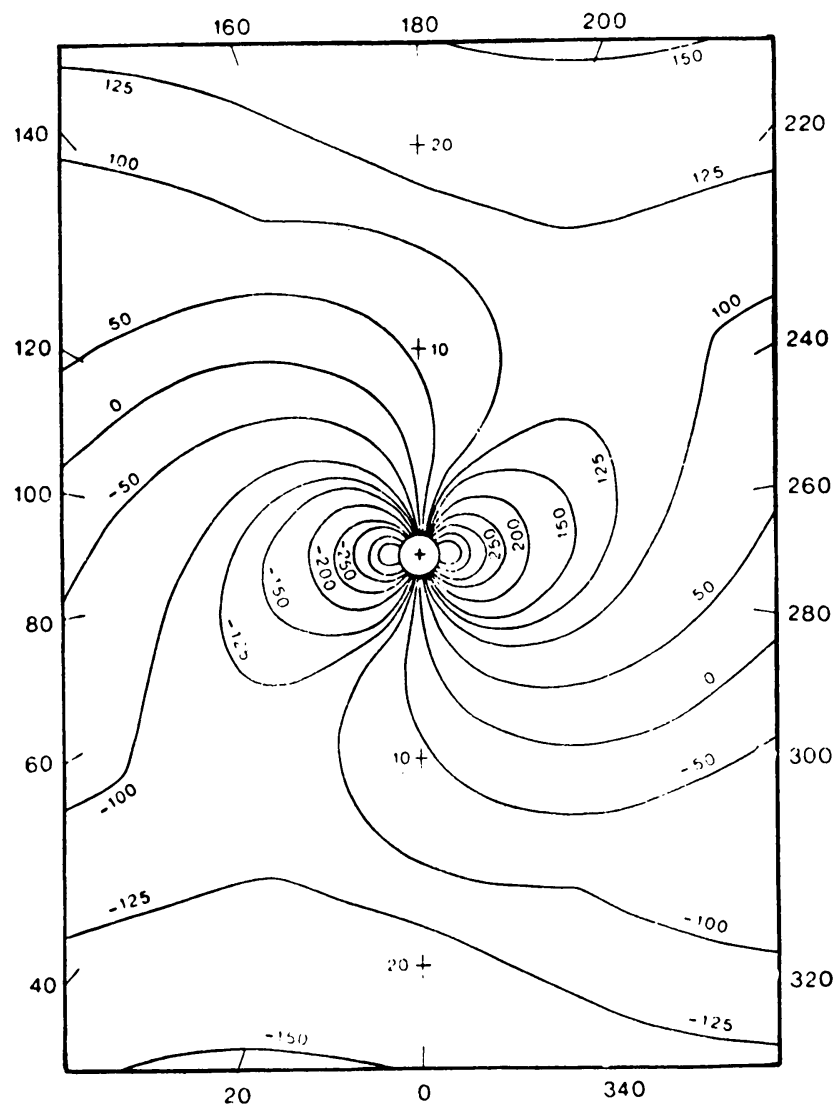

Fig. 3 - Radial-velocity contours in the equatorial plane of a star with an expanding envelope and an equatorial rotational velocity of $569 \mathrm{~km} / \mathrm{sec}$ (Poeckert and Marlborough, 1978a). The observer is at azimuthal angle zero where the angles are labelled around the outside of the figure. Contours are labelled in $\mathrm{km} / \mathrm{sec}$.

from Fig. 4 that the central polarization maximum is still present at $6 \mathrm{R}_{t}$ even though there is considerable line emission. It is apparent, therefore, that line absorption is more important than line emission (recall the observations of Eta Cen) in determining the line polarization. In retrospect this is not surprising since the continuum polarization, especially at the Balmer jump, is itself dominated by absorption effects. This means that the higher Balmer lines should also show decreases in polarization, even though very little emission is present. This is indeed the case (Mc Lean, 1981) and is the cause of the "rounding" of the abrupt polarization jump as the Balmer limit is approached. 


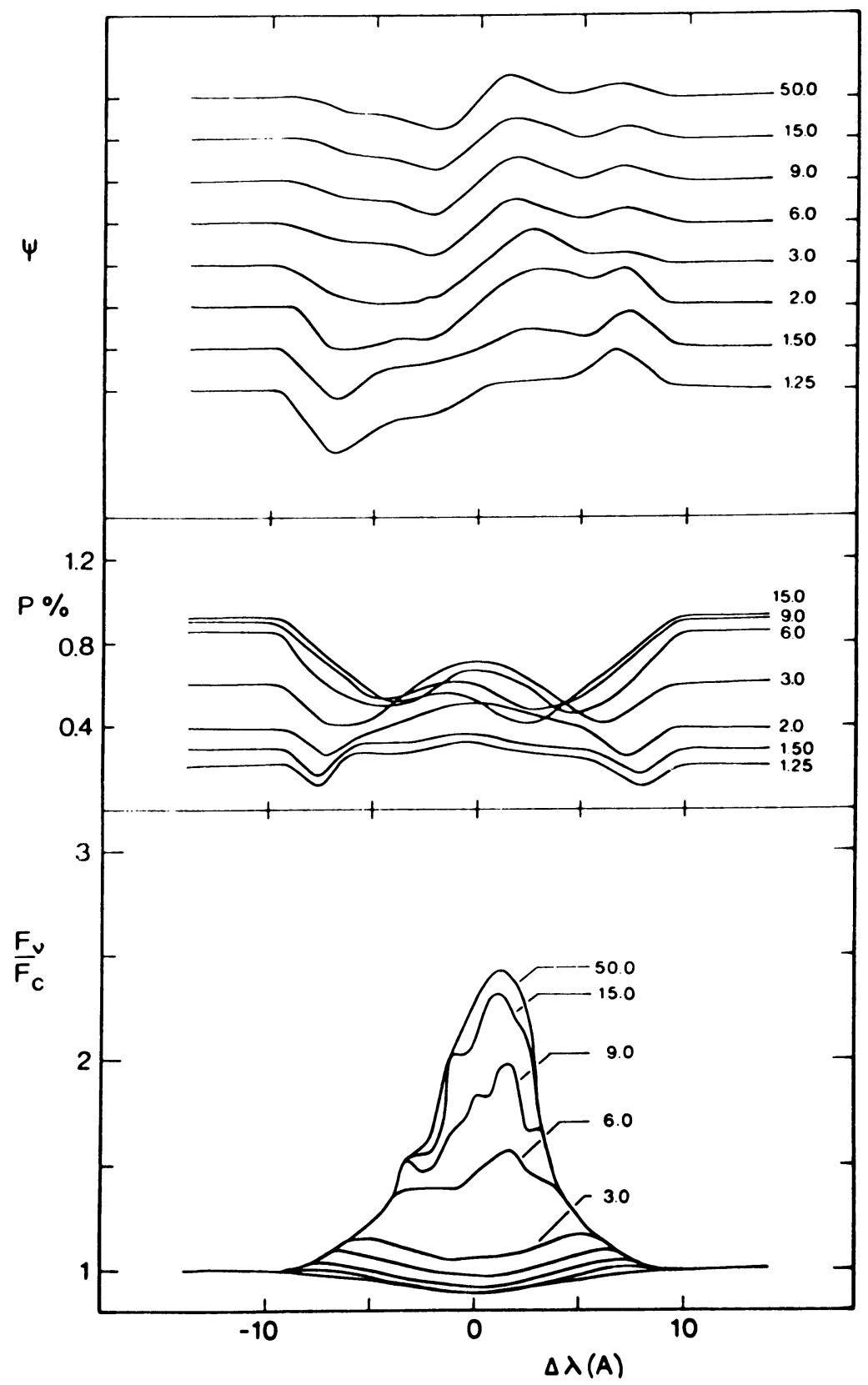

Fig. 4 - Position angle of polarization, percent polarization and line profile (top to bottom) derived by cutting off the transfer solution at the radial point in the envelope indicated by the number on each profile corresponding in Fig. 3 to the distance in stellar radii from the star's center (Poeckert and Marlborough, 1978a). 


\section{CONCLUSIONS}

Polarimetry has proven to be particularly valuable in our attempt to understand the nature of the extended envelopes about Be stars, since these envelopes are flattened due to the high rotational velocity of these stars. In particular the wavelength dependence of the polarization depends upon the electron density, the electron temperature and the size and shape of the envelope. Furthermore, detailed variations of polarization across atomic lines serve as a probe of the dynamics, particularly the rotational and expansion velocities, of the gas in these envelopes. Although significant advances in observations have been made, especially in the area of high-resolution spectropolarimetry, a great deal remain to be done observationally. Especially needed are systematic and detailed observations of more stars, since only four stars, Gamma Cas, Zeta Tau, $48 \mathrm{Lib}$ and Phi Per, have been observed in more than a routine manner. While there may have been significant progress in developing analytical models and impressive advances with stellar wind models, incorporating rotational and expansion velocities of the envelope gas, an explanation of the detailed spectral polarimetry observed across atomic lines will require more sophisticated models.

\section{REFERENCES}

Ange1, J.P.R. and Landstreet, J.D. : 1974, Ap.J. 191, 457

Beaver, E.A., McIlwain, C.E., Choisser, J.P., Wysoczanski, W. : 1972 Adv. Electronics and Electron Physics, 33B, 863

Brown, J.C. and McLean, Ian S. : 1977, Astron. Ap. 57, 141

Brown, J.C. and McLean, Ian, S. and Emslie, A.G. : 1978, Astron. Ap. 68,415

Capps, R.W., Coyne, G.V. and Dyck, H.M. : 1973, Ap.J., 184, 173

Cassinel1i, J.P. and Haisch, B.M.: 1974, Ap.J., 188, 101

Coyne, G.V. : 1976, in IAU Symposium 70, Be and Shell Stars, ed.

Slettebak, A. (Dordrecht : Reide1) p. 233

Coyne, G.V. and Kruszewski, A. : 1969, Astron. J., 74, 528

Coyne, G.V. and McLean, Ian S. : 1975, Astron. J., 80, 702

Danie1, J.Y. : 1980, Astron. Ap., 86, 198

Hayes, D.P. : 1978, Ap.J. 219, 952

Hazlehurst, J. : 1967, Zs. Ap., 65, 311

Huang, S.S. : 1972, Ap. J., 171, 549

Jones, T.L. : 1979, Ap.J. 228, 787

Kemp, J.C., Rieke, G.H., Lebofsky, M.J. and Coyne, G.V. : 1977, Ap. J. 215, L 107

McLean, Ian S. : 1977, Astron. Ap., 55, 347

McLean, Ian S.: 1979, M.N.R.A.S., 186, 265

McLean, Ian S. : 1980, in New Techniques in Stellar Photometry and Polarimetry, Ricercher Astronomiche, Vo1. 10, ed. McCarthy, M.F. (Specola Vaticana : Città del Vaticano), p. 71 
McLean, Ian S. : 1981, M.N.R.A.S. in press

McLean, Ian S. and Brown, J.C. : 1978, Astron. Ap., 69,291

McLean, Ian S. and Clarke, D. : 1976, IAU Symposium 70, Be and Shel1

Stars, ed. Slettebak, A. (Dordrecht : Reide1), p. 261

McLean, Ian S. and Clarke, D. : 1979, M.N.R.A.S., 186, 245

McLean, Ian S., Coyne, G.V., Frecker, Jack E. and Serkowski, K. : 1979, Ap. J. 228, 802

Miller, J.S., Robinson, L.B. and Schmidt, G.D. : 1980, P.S.A.P., 92,702

Nordh, H.L. and Olofsson, S.G. : 1977, Astron. Ap., 56, 117

Poeckert, R. : 1975, Ap. J., 196, 777

Poeckert, R., Bastien, P. and Lanstreet, J.D. : 1979, Astron. J., 84, 812

Poeckert, R. and Marlborough, J.M. : 1976, Ap. J., 206, 182

Poeckert, R. and Marlborough, J.M. : 1977, Ap. J., 218, 220

Poeckert, R. and Marlborough, J.M. : 1978a, Ap. J., 220, 940

Poeckert, R? and Marlborough, J.M. : 1978b, Ap. J. Supp1., 38, 229

Poeckert, R. and Marlborough, J.M. : 1979, Ap. J., 233, 259

Slettebak, A. : 1976, IAU Symposium 70, Be and Shel1 Stars (Dordrecht: Reide1)

Stockman, H.S., Angel, J.R.P. and Hier, R. : 1979, B.A.A.S., 10, 689

Tomaszewski, L., Landstreet, J.D. and Symonds, D. : 1980, in press. 


\section{DISCUSSION}

Stalio: Did you compute your radiative transfer problem in rotating and expanding atmospheres with Sobolev's approximation? If not, could you give a very brief description of the method?

Poeckert: No, we did not use the Sobolev approximation. We assume that the source function and opacity is a linear function of geometric distance between sample points along a line of sight. The sampling frequency depends on the density and velocity gradients along the line of sight. The phase is evaluated at 2000 frequencies within the line.

Peters: Is it not true that as a class Be-shell stars show the highest degree of polarization?

Coyne: As best I know it is not true that the largest polarizations are associated with the shell phases of Be stars or that only those stars which have shell phases (shell stars) have the largest polarizations.

Hirata: The elliptic ring-(or disk) model for the $V / R$ variables could be checked by the polarimetric observations. Is there any indication which supports or denies such model in the polarimetric observations?

Coyne: I know of no polarimetric observations that would exclude an elliptical ring model. Infact the elliptical ring model can be considered a specification of the more general scattering flattend envelope models being applied to explain the polarization of Be stars.

Sonneborn: I wish to comment on the net linear polarization of the flux from a rotating star. Model stellar atmospheres for rapidly rotating stars show the intrinsic polarization of the photospheric radiation is at most $.05 \%$ in the visual, but may be as large as $2.5 \%$, in the far ultraviolet. I will discuss this further on my talk on Friday.

de Freitas Pacheco: Could you comment on the observational fact you have mentioned that the polarization decreases on the line core and the result of model calculations.

Coyne: The polarization generally decreases to the line center due to the addition of unpolarized emission flux which dilutes the polarization. One cannot, however, exclude, infact in some cases we observe this, that the emission flux is partially polarized. A further effect is that in the presence of a central absorption core imposed on the emission line we detect an increase in polarization due, apparently, to the further absorption of the direct flux. 